\title{
Probing Material Morphology and Deformation as a Response to in situ Loading using X-ray Tomography
}

Brian M. Patterson ${ }^{1}$, Lindsey Kuettner ${ }^{1}$, Nikolaus Cordes $^{2}$, Kevin Henderson ${ }^{1}$, Matthew Herman ${ }^{1}$, Cindy Welch $^{1}$, John Carpenter ${ }^{1}$, Colt Montgomery ${ }^{1}$, Tao Sun $^{3}$, Kamel Fezzaa ${ }^{3}$, Xianghui Xiao ${ }^{3}$, Jason Williams ${ }^{4}$ and Nikhilesh Chawla ${ }^{4}$

1. Materials Science and Technology Division, Los Alamos National Laboratory, Los Alamos, NM, USA.

2. Idaho National Laboratory, Idaho Falls, ID, USA.

3. Advanced Photon Source, Argonne National Laboratory, Argonne, IL, USA.

4. Center for 4D Materials Science, Arizona State University, Tempe, AZ, USA.

Multi resolution (size and temporal) X-ray computed tomography (CT) is critical for understanding a materials morphology as a result of processing. Measuring the morphological features, (e.g. porosity, ligament diameter, separated phases) can give clues as to the optimal processing parameters. Features measured with X-ray tomography may be as small as submicrometer, to 100's of micrometers in size, internal to the sample, and are often used as a starting point for modeling. Additionally, in situ measurements, in which the material is subjected to a mechanical load during imaging [1] is often used to validate and test a material model's robustness. Due to its ability to collect information on a material's true microstructural geometry, CT is often used as a starting structure for 3D FEM [2-5], to examine the artifacts contained within 3D printed materials, and the damage within a material due to insult $[6,7]$.

For this presentation, we will discuss the challenges of using CT (both lab and synchrotron based) imaging and using it to answer questions relating processing and manufacturing to material performance. First, multi-scale CT of metal AM is used to better understand print quality. Meso-scale imaging is used to understand the surface roughness and dimensional accuracy. Micro-scale CT is used to measure the distribution and size of particle inclusions as well as un-sintered material and voids. Figure 1 shows the surface finish and the distribution of voids within a 3D printed metal. Additionally, we will describe the process of collecting and analyzing 4D data, outline the challenges, and describe some recent efforts. Examples to be highlighted include the deformation of 3D printed soft materials; tensile glass-nylon composite specimens, as well as acrylic-based microlattices in compression (Figure 2) using real-time using synchrotron CT. In situ imaging of the bending and deformation of ligaments provides a clear picture of the stress concentration and fracture within these materials, which is critical for model validation.

References:

[1] BM Patterson et al., in "Materials Discovery and Design By Means of Data Science and Optimal Learning", ed. T Lookman, S Eidenbenz, F Alexander, C Barnes, (Springer) p. 130.

[2] B Branch et al., Polymer 160 (2019), p. 325. doi:10.1016/j.polymer.2018.10.074

[3] JD Yeager et al., AIP Conference Proceedings (2018) p. 070033. doi:10.1063/1.5044842

[4] DJ Walters et al., International Journal of Mechanical Sciences 140 (2018), p. 151. doi:https://doi.org/10.1016/j.ijmecsci.2018.02.048

[5] D Tourret et al., Metallurgical and Materials Transactions A 48(11) (2017), p. 5529. doi:10.1007/s11661-017-4302-8 
[6] JCE Mertens et al., Journal of Materials Science 52(20) (2017), p. 12185. doi:10.1007/s10853-017$1339-4$

[7] MJ Herman et al., Fusion Science and Technology (2017), p. 1. doi:10.1080/15361055.2017.1387454
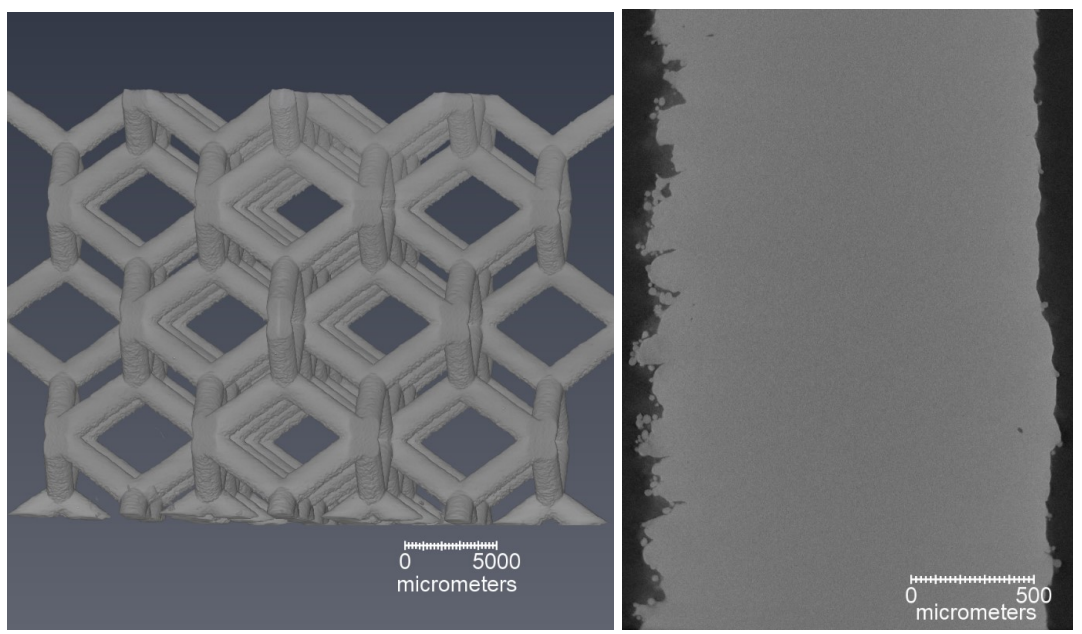

Figure 1. X-ray CT image of a 3D printed stainless steel lattice structure (left), and a high resolution image through the cross section highlighting both the variation in surface roughness as well as the presence of voids and inclusions.

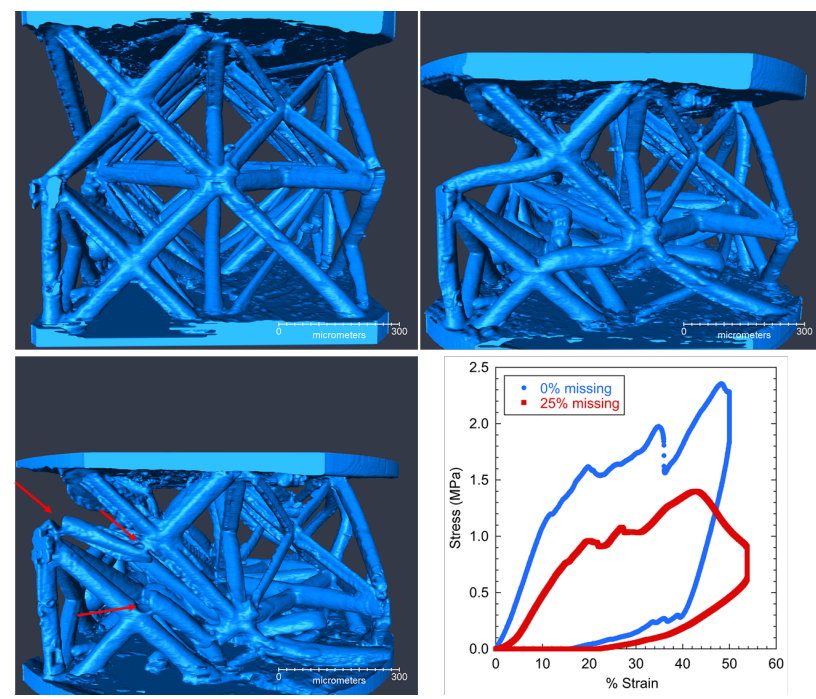

Figure 2. Sequence of 3D images of a microlattice taken during in situ compression. Stress strain curves also highlight the deformation and damage (as seen by the abrupt changes in response due to ligament buckle). 